\title{
Interface logicielle pour l'apprentissage des techniques d'imagerie médicale et des gestes médicochirurgicaux assistés par ordinateur
}

\author{
Th. Leloup \\ Université Libre de Bruxelles, Faculté des Sciences Appliquées, Laboratoire de l'Image : Synthèse et Analyse, \\ 50 av. Roosevelt, CP 165/57, B-1050 Bruxelles \\ Email : tleloup@ulb.ac.be
}

\begin{abstract}
RESUME : L’imagerie médicale et les gestes médicochirurgicaux assistés par ordinateur constituent deux disciplines importantes dans la formation des ingénieurs civils biomédicaux. Une interface logicielle permettant la mise en pratique des notions enseignées est décrite dans ce papier. Elle permet une mise en route rapide pour les étudiants tout en assurant une compatibilité avec une des principales bibliothèques logicielle du domaine.
\end{abstract}

Mots clés : Imagerie médicale, gestes médicochirurgicaux assistés par ordinateur, interface logicielle didactique.

\section{INTRODUCTION}

Les formations d'ingénieurs civils biomédicaux sont de plus en plus courantes dans les universités européennes. Ce domaine, pluridisciplinaire par essence, représente un véritable défi pour l'enseignant, devant maîtriser simultanément des notions de biologie, de médecine et d'ingénierie. L'enseignement des techniques d'analyse d'images médicales et des gestes médicochirurgicaux assistés par ordinateur constituent deux exemples particulièrement représentatifs dans la formation d'ingénieurs civils biomédicaux.

L'analyse des images médicales, permettant d'obtenir des paramètres pertinents pour le médecin à partir d'informations brutes (images numériques 2D ou 3D) est actuellement un domaine est en plein essor. Cette démarche s'inscrit tout à fait dans le cadre actuel de notre société où il faut extraire d'une multitude d'informations celles qui nous paraissent importantes. Du fait de la numérisation de plus en plus importante des images médicales, l'analyse de telles données constitue un support de choix pour la collaboration entre médecins et ingénieurs, permettant de comparer les techniques visuelles et automatiques.

Les gestes médicochirurgicaux assistés par ordinateur (GMCAO) nécessitent une très grande multidisciplinarité : non seulement ils imposent une collaboration étroite entre médecins et ingénieurs, mais ils font également appel à de nombreux domaines des sciences de l'ingénieur. Par ailleurs, il s'agit d'une discipline possédant un caractère très appliqué. Les GMCAO permettent en particulier de rendre les techniques chirurgicales moins invasives, en utilisant des modèles recalés avec le patient. Les implications au niveau socio-économiques sont nombreuses : chirurgie ambulatoire, réduction des durées d'hospitalisation, importantes économies dans les systèmes de soins de santé, etc. Bien acceptées par les patients, elles recèlent un important potentiel de croissance, notamment dans les systèmes d'instrumentation et de visualisation, les techniques d'imagerie et de recalage, les implants spéciaux, les composants microtechniques, etc.

Les notions fondamentales, enseignées habituellement lors de cours magistraux, peuvent bénéficier des récentes techniques multimédia permettant d'illustrer les principes développés dans le cadre de ces deux disciplines très visuelles. Toutefois, l'application de ces concepts théoriques à des problématiques concrètes permet à l'étudiant de mieux comprendre la matière enseignée et de développer des compétences pratiques telles que la résolution de problèmes multidisciplinaires ou le travail en équipe.

La mise en place de tels travaux pratiques exige une collaboration étroite avec le monde médical tant pour la détermination des sujets des travaux, que pour l'acquisition et l'interprétation des données. Les médecins sont souvent partie prenante de telles collaborations car elles débouchent fréquemment sur des projets de recherche communs. L'initiative peut également provenir du monde médical.

L'outil informatique, incontournable dans ce genre de disciplines, peut rebuter l'enthousiasme des étudiants à cause d'une maîtrise insuffisante de la programmation. Plusieurs bibliothèques logicielles existent dans ces domaines ; citons entre autres ITK [1], MITK [2,3] et IGSTK [4]. Malheureusement, elles sont assez difficiles à prendre en main et nécessitent à la fois un bon niveau de connaissances informatiques des étudiants et un temps d'adaptation important pour pouvoir être utilisées efficacement. Les logiciels standards de traitement d'image ne conviennent généralement pas à cause des formats spécifiques des images médicales (format DICOM [5] par exemple). Une connexion à d'autres dispositifs - comme par exemple un localisateur optique tridimensionnel - est souvent nécessaire dans le cadre des GMCAO.

Ce papier a pour but de présenter une interface logicielle innovante servant de base pour des projets d'analyse d'images médicales et permettant aux étudiants de se familiariser avec les techniques de GMCAO.

Pour les projets d'analyse d'images médicales, les étudiants concernés sont en première année de leur formation de Master Ingénieur Civil Biomédical (bac+4 à bac+5). Pour les GMCAO, ils sont en seconde année du même Master. Ils ont en principe acquis au fil des années précédentes les notions de base en $\mathrm{C}++$ et en 
programmation orientée objet.

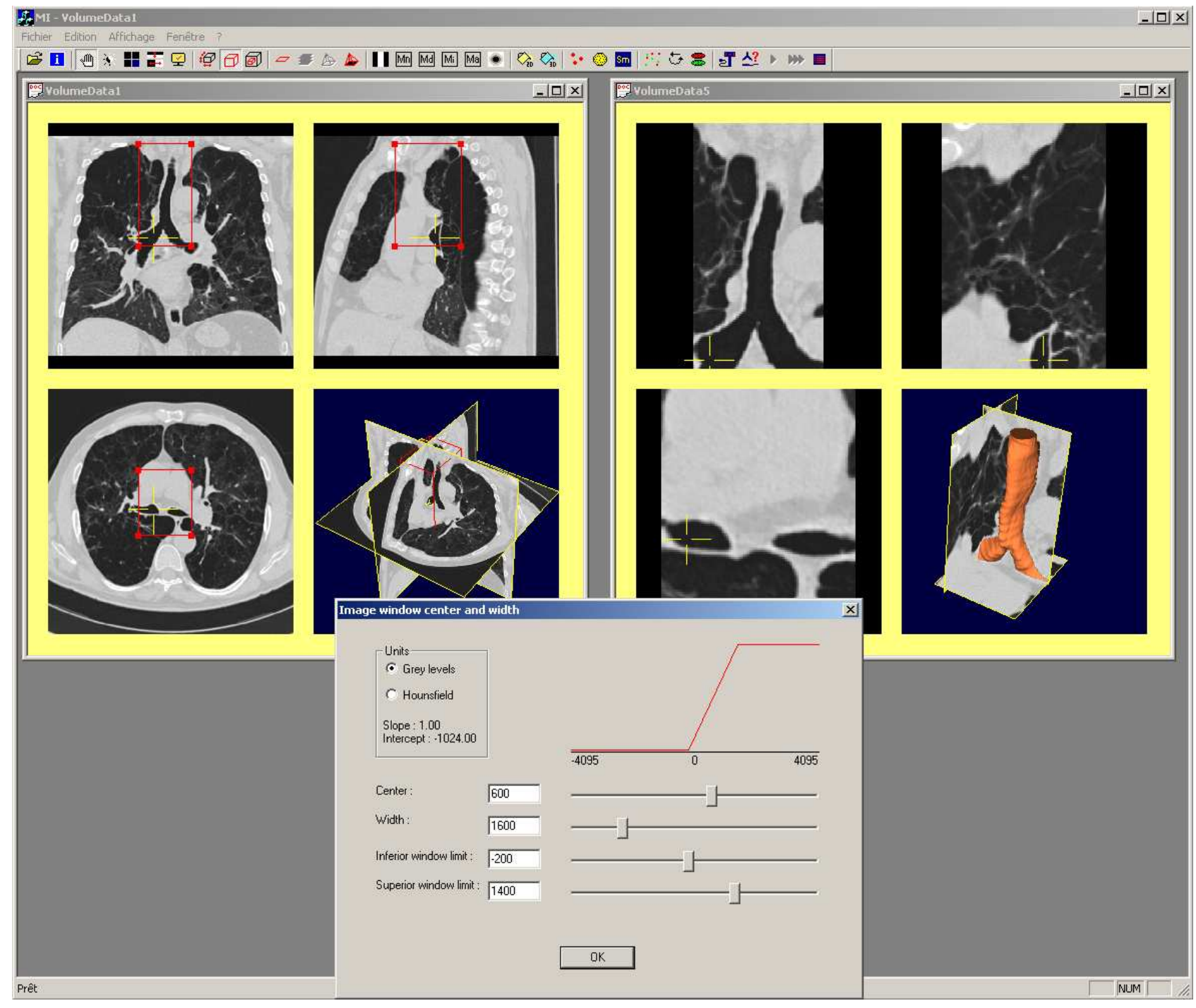

Fig. 1 : Interface logicielle illustrant différentes fonctionnalités implémentée : curseur synchronisé (croix jaune), sélection d'une zone d'intérêt (parallélépipède rectangle rouge), définition d'une nouvelle image $3 D$ selon cette zone (fenêtre de droite), reconstruction $3 D$ (orange) et modification de la visualisation des niveaux de grix (bôte de dialogue du bas).
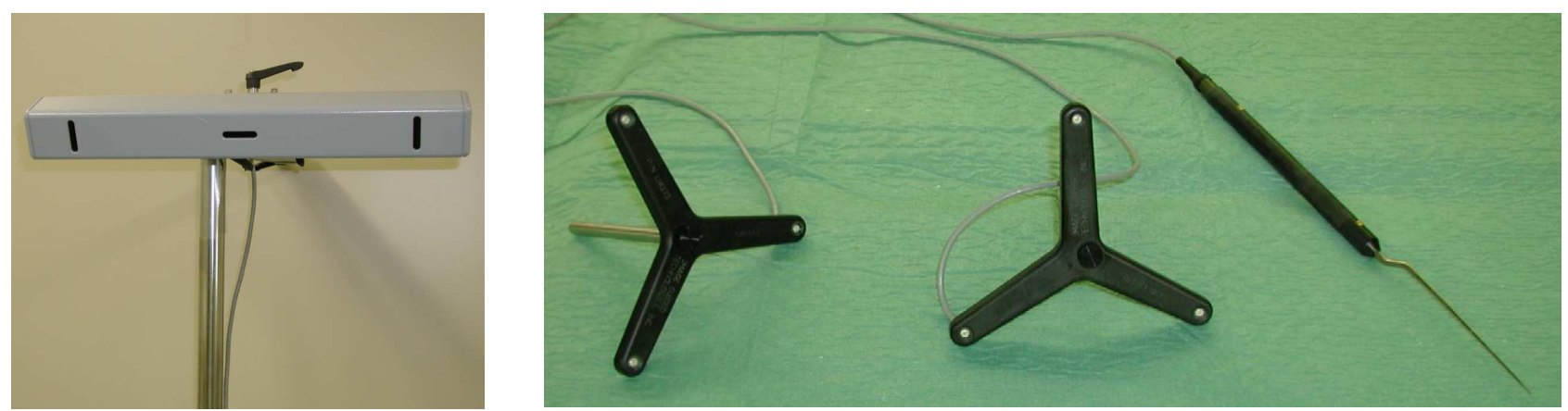

Fig. 2 : Localisateur optique (à gauche) constitué de 3 caméras linéaires et instruments (à droite) repérables par ce dernier à l'aide de diodes infrarouges intégrée. 


\section{PRESENTATION DE L'INTERFACE}

\subsection{Présentation générale}

Le logiciel est implémenté en $\mathrm{C}++$. Ce choix a été dicté d'une part par la volonté d'utiliser la bibliothèque ITK, largement reconnue dans le domaine du traitement d'images médicales, et d'autre part par le fait que tous nos étudiants ont appris ce langage en détail lors des premières années de leur formation. L'interface graphique utilisateur repose sur les Microsoft Foundation Classes (MFC), abondamment documentées, et sur la bibliothèque OpenGL pour l'affichage 3D. Le logiciel a uniquement été développé dans l'environnement de programmation Visual Studio 2005, disponible gratuitement pour les étudiants (version Express).

L'architecture du logiciel est du type Multiple Documents Interface: la fenêtre principale peut contenir plusieurs autres fenêtres, chacune d'entre elles étant relative à une image médicale tridimensionnelle au format DICOM. Ces images peuvent provenir de plusieurs modalités : tomodensitométrie (CT-scanner), imagerie par résonnance magnétique (IRM), tomographie par émission de positrons (TEP), etc. La fenêtre principale du logiciel permet de contrôler un localisateur optique 3D (FlashPoint 5000, Image Guided Technologies [6]) utilisé lors de chirurgies assistées par ordinateur et disponible au sein de notre laboratoire. La communication avec ce dispositif est assurée par une connexion série. Un convertisseur USB/série est utilisé sur les ordinateurs ne disposant plus de ce type de port.

\subsection{Imagerie médicale}

Chaque fenêtre correspondant à une image médicale 3D comporte quatre visualisations différentes des données : trois correspondant aux vues axiale, sagittale et coronale ; la quatrième correspondant à la visualisation des reconstructions tridimensionnelles obtenues à partir de l'image médicale. Ces quatre vues sont automatiquement synchronisées entre elles à l'aide d'un curseur 3D, dont la position peut être modifiée par l'utilisateur (Fig. 1).

Une boîte de dialogue interactive permet de modifier la fenêtre des niveaux de gris (centre et largeur). En effet, les images possèdent généralement plus de niveaux de gris (souvent 4096) que ceux affichables (souvent 256). Une fonction linéaire de correspondance entre les niveaux des images et ceux affichés peut être ajustée. Son effet peut être directement visualisé dans les quatre vues (Fig. 1).

Plusieurs opérations de base de traitement d'images sont implémentées: seuillage, filtres de voisinages (minimum, maximum, moyenne, médian), carte de distance, croissance de région en fonction d'un intervalle de niveaux de gris, etc. La réduction de l'image 3D à une zone parallélépipédique définie de façon interactive (cropping) est également disponible.
Le résultat peut être ouvert dans une seconde fenêtre sans perdre les données de la première (Fig. 1).

Une modélisation tridimensionnelle de structures anatomiques peut être effectuée en sélectionnant l'intervalle des niveaux de gris correspondants (algorithme du Marching Cube [7]). La simplification du maillage est également implémentée en fonction du nombre maximum de facettes souhaitées, permettant ainsi d'obtenir une visualisation tridimensionnelle fluide pour les gros modèles ou les ordinateurs moins performants. Plusieurs maillages $3 \mathrm{D}$ peuvent être reconstruits et visualisés simultanément. Le logiciel propose également des options au niveau des couleurs et de la transparence des modèles affichés.

Des points anatomiques peuvent être sélectionnés au sein des trois vues de l'image ou en cliquant sur un point du modèle surfacique 3D (picking). Ces points peuvent être enregistrés dans un fichier texte, utilisable ultérieurement au sein de ce programme ou d'autres logiciels.

Les données de l'image 3D sont stockées selon un format compatible avec ITK, rendant possible l'utilisation de toutes les fonctions disponibles dans cette bibliothèque. Cependant, les principales fonctions d'accès aux données de niveaux de gris ont été réimplémentées dans une librairie de fonctions simples, mieux documentées et facilement utilisable par les étudiants non familiarisés avec la bibliothèque ITK. Ceci revêt une importance capitale pour des travaux pratiques devant s'effectuer en quelques heures.

\subsection{GMCAO}

Dans le cadre des GMCAO, le traitement des images médicales est d'une grande importance. Les outils énoncés plus haut restent disponibles. En outre, les aspects de recalage d'images avec l'espace 3D du patient et l'utilisation d'instruments repérables en 3D (Fig. 2), par un localisateur optique par exemple, sont cruciaux.

Une fonction de recalage de paires de points correspondants est disponible au niveau du logiciel. La correspondance s'établit en fonction des noms attribués aux différents points. Ceux-ci doivent être identiques à l'exception d'un suffixe qui précise l'appartenance au modèle source ou cible. Les transformations rigides résultantes (rotations et translations) peuvent être enregistrées dans un fichier texte et/ou appliquées aux données sources. Des transformations rigides définies manuellement peuvent également être appliquées aux modèles 3D.

Le contrôle d'un dispositif de localisation 3D au sein de cette interface s'avère plus qu'utile pour développer des applications dans le cadre de cette discipline. L'acquisition des données de ce dispositif est implémentée au niveau du logiciel selon deux modes : mesures ponctuelles ou mesures continues. Pour chacun de ces modes, une fonction correspondante, pouvant être complétée par les étudiants, est appelée. Celle-ci permet d'interagir de façon ponctuelle ou continue avec 
l'image médicale et les mesures du localisateur3D. En outre ces dernières peuvent être visualisées et enregistrées sous format texte afin d'être traitées par d'autres logiciels (un tableur par exemple).

\section{UTILISATION PAR LES ETUDIANTS}

Notre interface convient aussi bien pour des travaux pratiques d'une demi-journée (environ 4 heures) que comme logiciel de base pour des projets plus ambitieux. Nous détaillons dans cette section les prérequis nécessaires à l'utilisation de l'interface et les objectifs pédagogiques poursuivis ainsi que quelques exemples d'applications en imagerie médicale et en GMCAO.

\subsection{Prérequis}

Pour les projets d'analyse d'images médicales, les prérequis nécessaires à l'utilisation de l'interface sont de deux types: d'une part, les connaissances concernant les méthodes d'analyse d'images médicales, et d'autre part, les connaissances informatiques indispensables à l'implémentation proprement-dite de ces techniques.

Pour les connaissances du premier type, une séance d'introduction concernant les bases de l'analyse d'images médicales (représentation des images, techniques de segmentation de base, modélisation...) est prodiguée aux étudiants. De plus, une série de livres de références est à leur disposition et permet d'approfondir certains domaines, en fonction de leur intérêt et du sujet de leur projet.

Les connaissances du second type sont, en principes, toutes acquises lors des années précédentes de leur formation (programmation $\mathrm{C}++$ orientée objet). Certains rappels sont parfois requis pour surmonter les difficultés de compréhension des bibliothèques logicielles.

Les travaux pratiques de GMCAO constituent l'occasion d'appliquer les méthodes vues précédemment lors du cours théorique; il suffit donc de rappeler au début de la séance le principe des techniques spécifiques concernées.

Sur le plan informatique, l'interface logicielle comprend déjà une série de fonctionnalités «vides » que les étudiants doivent implémenter au sein de fonctions bien précises. Ceci permet d'éviter qu'ils passent trop de temps sur des détails d'implémentation informatiques.

\subsection{Objectifs pédagogiques}

Les objectifs pédagogiques sont multiples. Outre la mise en pratique des connaissances acquises lors des années précédentes (essentiellement dans les domaines de l'informatique, de la géométrie, de l'analyse et de la physique), les projets d'imagerie médicale permettent également à l'étudiant d'apprendre à se servir d'ouvrages de référence spécialisés, de rechercher des informations pertinentes auprès de spécialistes médicaux, de communiquer avec ceux-ci en adoptant un langage commun, de se familiariser et d'utiliser des bibliothèques logicielles importantes dans ce domaine.

\subsection{Applications en imagerie médicale}

\section{a) Modélisation tridimensionnelle de structures osseuses}

Cet exercice permet aux étudiants de se familiariser avec les principales fonctionnalités implémentées dans le logiciel en seulement quelques heures. Ils y découvrent le choix d'un seuil des niveaux de gris pour segmenter une image, la sélection d'une structure anatomique particulière par croissance de région, l'obtention d'une modélisation 3D de cette structure et l'optimisation de sa visualisation.

Afin de faciliter cette première approche pratique de l'imagerie médicale, nous avons choisi des structures anatomiques simples à modéliser (i.e. les os) à partir de la modalité d'imagerie la plus appropriée (CT-scanner). Plusieurs examens de différentes régions anatomiques du corps humain sont disponibles pour les différents groupes (de 2 à 4 étudiants).

\section{b) Reconstruction d'une radiographie digitale}

A partir d'une image 3D tomodensitométrique, il était demandé aux étudiants de reconstruire une image radiographique, connaissant la position du plan de projection et celle de la source de rayons X. Le temps alloué à l'exercice était d'environ 4 heures, ce qui est relativement court étant donné la nature du problème.

L'organisation de la documentation des fonctions disponibles pour la programmation de l'exercice a été brièvement présentée aux étudiants durant la première heure.

Cette application a nécessité de la part de l'enseignant l'implémentation du positionnement interactif du plan et de la source de la radiographie au sein de notre logiciel (Fig. 3). Un bouton dans la barre d'outils permet d'appeler la fonction à compléter par les étudiants et à laquelle sont transmis les paramètres nécessaires au calcul de la radiographie digitale reconstruite. Cette dernière est ensuite ouverte automatiquement dans une autre fenêtre.

Outre les aspects mathématiques, physiques et informatiques intervenant dans cet exercice, l'étudiant a la possibilité de choisir lui-même la zone pour laquelle il veut reconstruire une radiographie, impliquant ainsi une démarche active et motivante. De plus, il peut aisément valider l'exactitude de l'image calculée. Enfin, plusieurs types de projections (radiographie classique, projection du maximum d'intensité, pondération par la profondeur etc.) peuvent être envisagés pour agrémenter l'exercice (Fig. 3). Ceci illustre bien les avantages de la reconstruction digitale de radiographies [8].

Cette interface logicielle a également été utilisée par les étudiants comme base pour le développement d'applications plus complexes dans le cadre de projets d'année plus ambitieux (7 ECTS) ou de mémoires de 
fin d'études. Citons par exemple la segmentation de tumeurs cérébrales par contours actifs ou la mesure de paramètres locaux de l'arbre bronchique.
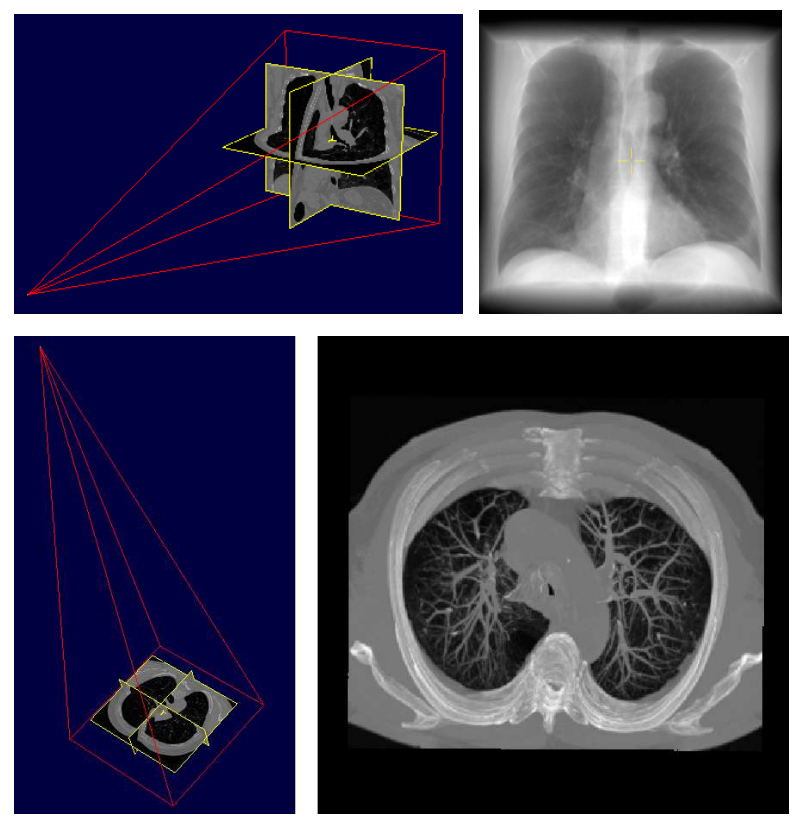

Fig 3: Reconstruction digitale d'une radiographie antéropostérieure des poumons (en haut) et d'une projection axiale du maximum d'intensité (en bas) ; la position de la source virtuelle de rayons x est représentée par le sommet de la pyramide rouge et le plan de projection par sa base carrée.

\subsection{Applications en GMCAO}

Les travaux pratiques du cours de GMCAO sont donnés sur plusieurs demi-journées. Chacune d'entre elles est dédiée à la réalisation d'un mini-projet concret et étroitement encadré.

\section{a) Recalage rigide de modèles de vertèbre}

Deux modèles de vertèbre exprimés dans des référentiels différents sont fournis aux étudiants (Fig. 4). On leur demande de recaler leur surface extérieure par l'algorithme Iterative closest point (ICP) à implémenter dans une fonction vide recevant les deux maillages en paramètres. Pour recaler les deux modèles, ils doivent utiliser la fonction de recalage de paires de points correspondants qui est déjà implémentée. La visualisation du résultat après chaque itération ajoute un aspect dynamique motivant fortement les étudiants.

\section{b) Conception d'un clavier virtuel}

Les interactions entre le chirurgien et les systèmes informatiques ne peuvent s'effectuer à l'aide d'une souris et d'un clavier grands publics pour des raisons de stérilité. Une interface homme-machine stérilisable doit donc être proposée au chirurgien. Elle peut par exemple être un clavier virtuel, plaque métallique gravée de touches et fixée rigidement à un référentiel constitué de marqueurs (diodes infrarouges) repérables par le localisateur optique 3D. Le chirurgien peut sélectionner un bouton en le touchant avec l'extrémité d'un pointeur stérile, également localisé en 3D. Un tel dispositif est très facile à fabriquer et peut être utilisé par exemple pour contrôler la visualisation d'un modèle 3D. L'implémentation logicielle de cette interface comprend plusieurs étapes : calibrage du clavier virtuel à l'aide du pointeur (définition de la position des touches virtuelles par rapport au repère qui lui est fixé), suivi de la position du pointeur par rapport au repère fixé au clavier, détection des touches virtuelle sélectionnées par le pointeur (génération d'événements) et réponse aux différents événements détectés.

Pour ce mini-projet, les étudiants doivent donc compléter les deux fonctions de mesures ponctuelles et continues du localisateur optique.
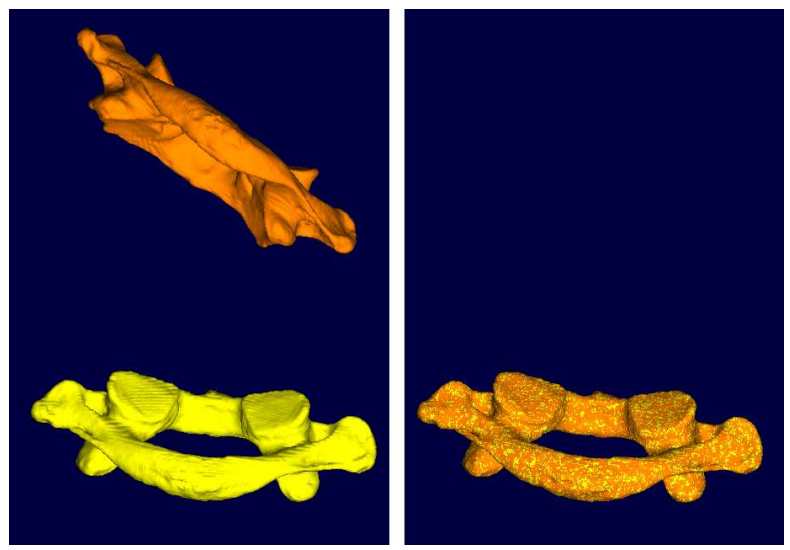

Fig 4: Recalage de deux modèles de vertèbres par la méthode ICP. Le résultat du recalage est illustré sur la figure de droite.

\section{c) Navigation sur un fantôme lombaire}

$\mathrm{La}$ réalisation de ce mini-projet constitue l'aboutissement des travaux pratiques de GMCAO puisqu'il s'agit de concevoir un système de navigation permettant le suivi d'un instrument (i.e. le pointeur) au voisinage d'un fantôme lombaire simulant l'anatomie d'un patient. Un examen CT-scanner de ce fantôme a été réalisé préalablement pour ce travail. Les étudiants doivent dans un premier temps modéliser en 3D les structures osseuses imagées. Ensuite, le recalage entre le fantôme et le modèle virtuel doit être réalisé sur base d'au moins trois paires de points correspondants. Ceuxci sont sélectionnés avec la souris sur le modèle informatique et digitalisés à l'aide du pointeur sur le fantôme lombaire, dans un repère fixé à ce dernier. Le suivi continu du pointeur peut alors être affiché sur l'image 3D reconstruite en réalité virtuelle (Fig. 5). De plus, les trois coupes orthogonales dans l'image tomodensitométrique peuvent être synchronisées sur l'extrémité de l'instrument.

Outre les performances techniques pour réaliser le système de navigation, ce mini-projet permet également aux étudiants de se mettre à la place du chirurgien en se 
servant du système de réalité virtuelle qu'ils viennent de réaliser. Ils peuvent ainsi tester leur coordination manuelle et visuelle, par exemple pour positionner le pointeur dans l'axe d'un pédicule vertébral, simulant l'insertion de vis destinées à traiter certaines pathologies rachidiennes.

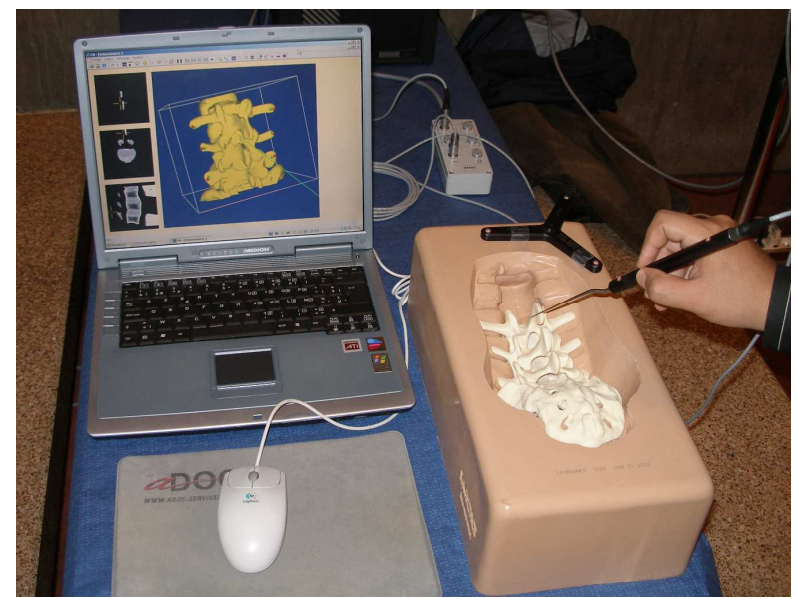

Fig 5: Guidage du positionnement $d u$ pointeur sur le fantôme lombaire par réalité virtuelle : l'axe de l'extrémité de l'instrument est illustré par un segment de droite sur la vue $3 D$ du PC.

\section{IMPACT PÉDAGOGIQUE SUR LA FORMATION}

Les projets d'imagerie médicale étant de plus longue haleine (ils se déroulent sur les trois premiers quarts de l'année académique), leurs impacts sur la formation sont multiples : travail de recherche préparant au mémoire de l'année suivante, apprentissage de la communication avec des experts médicaux de formation scientifique différente (établissement de relations de travail efficaces), occasion de rédiger un document sérieux et convaincant et d'en faire une présentation orale.

En ce qui concerne les travaux de GMCAO, l'impact pédagogique majeur reside dans prise de conscience de la difficulté de conception d'outils de guidage pour la chirurgie, conviviaux et simples d'utilisation. Les étudiants ont également la possibilité de se mettre à la place du chirurgien, qui doit réaliser un geste précis et complexe, en travaillant sur des fantômes anatomiques.

\section{CONCLUSION}

Le logiciel présenté dans ce papier permet à l'étudiant de s'initier à deux disciplines difficiles à mettre en pratique: l'imagerie médicale et les gestes médicochirurgicaux assistés par ordinateur. Les efforts nécessaires à la maîtrise de ses principales fonctionnalités restent raisonnables par rapport à ceux requis pour l'apprentissage des bibliothèques existantes dans le domaine. Ce logiciel peut être utilisé comme application à part entière mais aussi comme base à des développements didactiques ou des projets plus ambitieux. Pour ces derniers, la compatibilité avec une des principales bibliothèques d'imagerie médicale augmente encore les possibilités de notre plateforme. Les étudiants peuvent implémenter des fonctionnalités avancées en $\mathrm{C}++$, un langage qu'ils ont tous appris à maîtriser durant leurs premières années de formation et dont ils peuvent ainsi conserver la pratique.

Une version multiplateforme, utilisant par exemple la bibliothèque $Q t$ [9] pour la conception de l'interface graphique, doit encore être implémentée ultérieurement afin de permettre son déploiement sur tous les systèmes d'exploitation utilisés par les étudiants.

\section{Références}

[1] http://www.itk.org/

[2] http://www.mitk.net/

[3] http://www.mitk.org/

[4] http://www. igstk.org/

[5] http://medical.nema.org/

[6] http://www.imageguided.com/

[7] W. E. Lorensen, H. E. Cline, "Marching Cubes: A High Resolution 3D Surface Construction Algorithm", Computer Graphics (Proceedings of SIGGRAPH '87), Vol. 21, No. 4, pp. 163-169, 1987.

[8] T. Rohlfing, D. B. Russakoff, M. J. Murphy, C. R. Maurer, " An intensity-based registration algorithm for probabilistic images and its application for 2-D to 3-D image registration", Progress in biomedical optics and imaging, Vol. 3 (1), No. 22, pp. 581-591, 2002.

[9] http://trolltech.com/products/qt/ 